International Journal of Food Science, Nutrition and Dietetics (IJFS)

ISSN 2326-3350

\title{
Development and Evolution of Antioxidant Rich Fruit Beverage
}

Research Article

Dharti $\mathrm{P}^{1}$ and Dhvanika $\mathrm{P}^{2 *}$

${ }^{1}$ Smt. K.P.Patel college of Home Science, Anand People's Medicare Society ,Anand 388001, Gujarat, India.

${ }^{2}$ Professor Smt. K.P.Patel college of Home Science, Anand People's Medicare Society ,Anand 388001, Gujarat, India.

\section{Abstract}

The purpose of this study was to developed and analyzes Antioxidant rich fruit beverages to determine antioxidant concentrations. It may provide protection differently against oxidative stress because they are different in antioxidant capacity.Fruits are used commonly in form of fleshy or pulpy, juice. Fruit juices and beverages are most effective against disease related to such as reduced levels of oxidative stress, obesity, diabetes, atherosclerosis, high blood pressure, diabetes, osteoporosis, arthritis, some forms of cancer and even biological aging, chronic inflammation, Cardiovascular diseases. Antioxidant analysis such as Total Phenol, Flavanoids, Total antioxidant capacity, Vit C, Anthocyanins content in Pomegranate, strawberry, Red grapes, orange, Dried Green Tea. Fruit beverages were prepared at different ratio. Its' content of antioxidants shows highly significant different with each other.

Keywords: Antioxidant, Fruit beverages, Fruit juice, Total phenol, Flavanoid

\section{*Corresponding Author:}

Dhvanika Patel,

Smt. K.P.Patel college of Home Science, Anand People's Medicare Society, Anand 388001, Gujrat, India.

Tel: +91-9979095023

E-mail: dhvanikunj@yahoo.com

Received: Julu 14, 2014

Accepted: October 07, 2014

Published: October 14, 2014

Citation: Dharti P and Dhvanika P (2014) Development and Evolution of Antioxidant Rich Fruit Beverage. Int J Food Sci Nutr Diet. 3(9), 160163. doi: http://dx.doi.org/10.19070/2326-3350-1400030

Copyright: Dhvanika $\mathbf{P}^{\odot}$ 2014. This is an open-access article distributed under the terms of the Creative Commons Attribution License, which permits unrestricted use, distribution and reproduction in any medium, provided the original author and source are credited.

\section{Introduction}

Fruits and vegetables are important sources of various vitamins, minerals, fibers, antioxidants, including ascorbic acid, carotenoids, and polyphenolic [1]. In common usage of fruits in the form of fleshy or pulpy, juicy, and it's usually sweet, with fragrant aromatic flavors. [2] Citrus fruits are rich in bitter limondsand vitamins such as ascorbic acid, carotenoid, folates, polyphenols, and flavonoids. Fruit juice is very similar to that of the original fruit and $100 \%$ natural product. Fruit juices are free from chemical preservatives and other additives. Fruit juices contain nutrients, such as minerals, trace elements, vitamins and phytochemicals, micronutrients, potassium, calcium and magnesium, low in sodium and fat. [4] A large variety of vitamin enhanced waters (VEWs) on the market and some products advertise high concentrations of antioxidants and therefore high antioxidant capacity. There are also the four berry juices that are thought to be rich in polyphenols: These are noni, goji, mangosteen and acai. Other widely available polyphenol rich beverages are fruit and berry juices, i.e., blueberry, mango, pineapple, etc. Some of these juices have been analyzed for their antioxidant content [5].
Phenolic compounds as secondary metabolites are widely present in fruits and vegetables. A positive relationship between consumption of food with abundant phenolics and low incidence of degenerative diseases, including cancer, heart disease, inflammation, arthritis, brain dysfunction and cataracts. [6] Flavonoids are diphenylpropanes that commonly occur in plants $>4000$ flavonoids have been identified. Which are flavones, flavanones, anthocyanins, flavans, and proanthocyanidins.Anthocyanins are a group of flavonoids in fruits such as blackberry, blueberry, cranberry, raspberry, strawberry, and boysenberry (Rubus sp.), range from 200 to $4950 \mathrm{mg}$. $\mathrm{kg}^{-1}$ of fresh fruit. Consumption of 100 to 150 $\mathrm{g}$ (-1cup) of these fruits could easily result in an intake of 100 to $200 \mathrm{mg}$ of anthocyanins [7].

\section{Materials and Methods}

In the present study was analyzed antioxidant contain from DGT, Orange, Pomegranate, Red grapes, Strawberry and Developed Fruitbevrage.Four samples was prepared in the form of different ratio mixing with water and evolution by sensory score and selected sample analysis of their anti-oxidant concentration.

\section{Total Phenol by FolinCioculten method}

Sample Preparation is $10 \mathrm{gm}$ of sample was mixed with $25 \mathrm{ml} 80 \%$ methanol, shaker for $2 \mathrm{hr}$, centrifuged at $8000 \mathrm{rpm}$ for $10 \mathrm{~min}$, collected supernatant. Standard of Gallic acid working solution $(20-100 \mu \mathrm{g})$ and made up volume $8 \mathrm{ml}$ with distilled water, $0.5 \mathrm{ml}$ of folincioculten (1:1) After $1 \mathrm{~min}$ add $1.5 \mathrm{ml}$ of sodium carbonate solutions, incubated for 2 hours at dark place and the reading $765 \mathrm{~nm}$ on UV Spectrophotometer. For Sample, $0.1 \mathrm{ml}$ of extracted sample and blank $8 \mathrm{ml}$ of $\mathrm{D} / \mathrm{W}$ was taken and treated same as standard.

\section{Flavonoid by using Kosalecet et al method}

Extracted Total phenol sample $20 \mathrm{ml}$ evaporated in a water bath, add $2 \mathrm{ml}$ of $80 \%$ of methanol. Standard working solution of 
catchin $(20-100 \mu \mathrm{g}), 4 \mathrm{mlD} / \mathrm{w}, 0.3 \mathrm{ml}$ Sodium nitrite, Incubated for 5 minutes, $0.3 \mathrm{ml}$ of aluminumtrichloride, $2 \mathrm{ml}$ Sodium hydroxide, volume made by the Distil water and read at $510 \mathrm{~nm}$ on UV Spectrophotometer. For sample take $1 \mathrm{ml}$ and blank $1.5 \mathrm{ml}$ of $\mathrm{D} / \mathrm{W}$ and treat same as standard.

\section{Anthocyanin Pigment Content by AOAC Official Method}

The maximum Extracted sample Solution portion added should be $10 \mathrm{ml}$ (1 part test portion, 4 parts buffer) so as not to exceed the buffer capacity. Sample Solution diluted (Mixed) with $\mathrm{pH} 1.0$ buffer and same sample diluted with $\mathrm{pH} 4.5$ buffer read at both 520 and $700 \mathrm{~nm}$. The diluted sample portions are read versus a blank with distilled water.

Note: - Measure observance within 20-50 min of preparation. If the diluted test portion is excessively turbid, clarify by centrifuging or filtering before measurement.

\section{Total Antioxidant Capacity by Macue}

Extracted Sample $0.5 \mathrm{ml}$ add $0.5 \mathrm{ml}$ Methanol, $3 \mathrm{ml} \mathrm{DPPH}$ reagent, incubated at $37^{\circ} \mathrm{C}$ for 20 minutes, measured at $517 \mathrm{~nm}$. The control sample was DPPH alone. Methanol was used as blank. For standardization Gallic acid use. Not DPPH Dye should be use after reach $\mathrm{OD} \leq 1$

\section{Vitamin-C by using Roe and Kurther}

Sample preparation takes 2 gm sample, add $10 \mathrm{ml}$ of $4 \%$ oxalic acid, centrifuge for 10-15 minutes. Collect the supernatant make up the volume $25 \mathrm{ml} \mathrm{4 \%}$ oxalic acid and treat with a pinch of charcoal, Standard (Ascorbic acid) working $10 \mathrm{ml}$, blank $10 \mathrm{ml}$ of $4 \%$ oxalic acid and sample $10 \mathrm{ml}$ titrate it against dye 2-6 dichloroindophenolblue, end point pink color.

\section{Development Of Fruit Beverage}

Fruit beverage was developed from pure fruit juice and DGT (dried green tea) Extraction (2 GM of DGT in $100 \mathrm{ml}$ of water boiled for 15-20mins), after that Fruit beverages was evaluation of antioxidant content. For the preparation of fruit beverage all required ingredients were purchased from the local market of
Anand.

\section{Standardization of Fruit Beverage}

The Fruit Beverages developed from DGT, Orange, Pomegranate, Red grapes, Strawberry, water were mixed in 5 different ratios as mentioned below. Sensory evaluation was done by 10 trained staff a member of the APMS campus Anand and one ratio (S4) was selected for further evolution. Sensory was done by composite scorecard for selection of fruit beverage. In sensory score card include colour, flavour, consistency, taste, after taste, astringency, over all acceptability, absent of defect.

Dehydrated Green Tea $=$ DGT, Orange $=\mathrm{O}$, Pomegranate juice $=$ $\mathrm{P}$, Red grapes $=\mathrm{RG}$, Strawberry juice $=\mathrm{S}$, Water $=\mathrm{W}$.

$\mathrm{S} 1: 10 \mathrm{ml} \mathrm{GT}+15 \mathrm{ml} \mathrm{O}+15 \mathrm{ml} \mathrm{P}+15 \mathrm{ml} \mathrm{RG}+15 \mathrm{ml} \mathrm{S}+30 \mathrm{ml} \mathrm{W}$ $\mathrm{S} 2: 10 \mathrm{ml} \mathrm{GT}+10 \mathrm{ml} \mathrm{O}+20 \mathrm{ml} \mathrm{P}+10 \mathrm{ml} \mathrm{RG}+20 \mathrm{ml} \mathrm{S}+30 \mathrm{ml} \mathrm{W}$ $\mathrm{S} 3: 10 \mathrm{ml} \mathrm{GT}+20 \mathrm{ml} \mathrm{O}+10 \mathrm{ml} \mathrm{P}+20 \mathrm{ml} \mathrm{RG}+10 \mathrm{ml} \mathrm{S}+30 \mathrm{ml} \mathrm{W}$ $\mathrm{S} 4: 20 \mathrm{ml} \mathrm{GT}+15 \mathrm{ml} \mathrm{O}+20 \mathrm{ml} \mathrm{P}+15 \mathrm{ml} \mathrm{RG}+20 \mathrm{ml} \mathrm{S}+10 \mathrm{ml} \mathrm{W}$ S 5: $20 \mathrm{ml} \mathrm{GT}+20 \mathrm{ml} \mathrm{O}+15 \mathrm{ml} \mathrm{P}+20 \mathrm{ml} \mathrm{RG}+15 \mathrm{ml} \mathrm{S}+10 \mathrm{ml} \mathrm{W}$

\section{Result and Discussions}

Total phenol, Flavanoid, Vitamin C, Anthocyanin, Total Antioxidant Capacity content of Strawberry, Pomegranate, Red grapes, Orange, DGT which were found highly significant difference $(* * \mathrm{P}<0.001)$ between comparisons to each other. (Table no 1.) where as Graphical show the DGT contain higher amount of Total phenol, Flavanoid, Vitamin C, and Pomegranate were contained higher amount of The Anthocyanin and The Total Antioxidant Capacity. (Fig 1) Fruit beverage was contained all antioxidants with good amount. (Table no 2). The sensory score for color, flavor, consistency, taste, aftertaste, Astringency, absence of defect, overall acceptability, was the highest score observed in Sample 4. (Table no. 3) and (Fig 2)

\section{Conclusion}

Antioxidants have become a recent interest to health conscious individuals. As a result, beverages containing high concentrations of Antioxidants are becoming increasingly popular. This is due to the health benefits that accompany polyphenol consumption, derived from their antioxidant properties.Antioxidant has been

Table 1. Total phenol, Flavanoid, Vitamin C, Anthocyanin, Total Antioxidant Capacity content of Strawberry, Promogrent, Red grapes, Orange, DGT

\begin{tabular}{|c|c|c|c|c|c|}
\hline Sample & Total phenol mg \% & Flavonoid mg \% & Vitamin C mg \% & Anthocyanin mg \% & $\begin{array}{l}\text { Total antioxidant } \\
\text { Capacity mg \% }\end{array}$ \\
\hline Strawberry & $\begin{array}{l}151.8^{\mathrm{b}} \\
\pm 8.743\end{array}$ & $\begin{array}{l}79.2667^{\mathrm{b}} \\
\pm 0.24037\end{array}$ & $\begin{array}{l}66.6667^{c} \\
\pm 1.66667\end{array}$ & $\begin{array}{l}16.2^{c} \\
\pm 0.11547\end{array}$ & $\begin{array}{l}83.1733^{c} \\
\pm 0.87027\end{array}$ \\
\hline Promogrent & $\begin{array}{l}158.927 \\
\pm 3.32306 \\
\end{array}$ & $\begin{array}{l}74.7333^{\mathrm{b}} \\
\pm 2.23035 \\
\end{array}$ & $\begin{array}{l}16.6667^{\mathrm{a}} \\
\pm 1.66667 \\
\end{array}$ & $\begin{array}{l}32.4^{\mathrm{d}} \\
\pm 0.1 \\
\end{array}$ & $\begin{array}{l}89.8^{c} \\
\pm 1.01489 \\
\end{array}$ \\
\hline Red grapes & $\begin{array}{l}113.1333^{\mathrm{ab}} \\
\pm 4.54252 \\
\end{array}$ & $\begin{array}{l}9.6^{a} \\
\pm 1.37477 \\
\end{array}$ & $\begin{array}{l}11.6667^{\mathrm{a}} \\
\pm 1.66667 \\
\end{array}$ & $\begin{array}{l}0.3367^{\mathrm{b}} \\
\pm 0.05207 \\
\end{array}$ & $\begin{array}{l}37.9333^{\mathrm{b}} \\
\pm 1.73622 \\
\end{array}$ \\
\hline Orange & $\begin{array}{l}76.3667^{\mathrm{a}} \\
\pm 0.81104 \\
\end{array}$ & $\begin{array}{l}7.1067^{\mathrm{a}} \\
\pm 0.10975 \\
\end{array}$ & $\begin{array}{l}43.3333^{\mathrm{b}} \\
\pm 1.66667 \\
\end{array}$ & $\begin{array}{l}0.2367^{\mathrm{ab}} \\
\pm 0.00667 \\
\end{array}$ & $\begin{array}{l}17.1167^{\mathrm{a}} \\
\pm 3.64398 \\
\end{array}$ \\
\hline Green tea & $\begin{array}{l}2446.9^{c} \\
\pm 47.69036\end{array}$ & $\begin{array}{l}226.0333^{c} \\
\pm 19.8696\end{array}$ & $\begin{array}{l}136.6667^{d} \\
\pm 3.33333\end{array}$ & $\begin{array}{l}0.05^{\mathrm{a}} \\
\pm 0.001\end{array}$ & $\begin{array}{l}39.6^{\mathrm{b}} \\
\pm 9.16642\end{array}$ \\
\hline F value & $\begin{array}{l}2264.413^{* *} \\
\pm 0.000\end{array}$ & $\begin{array}{l}98.390^{* *} \\
\pm 0.000\end{array}$ & $\begin{array}{l}578.750^{* *} \\
\pm 0.000\end{array}$ & $\begin{array}{l}39660.218^{* *} \\
\pm 0.000\end{array}$ & $\begin{array}{l}48.449^{* *} \\
\pm 0.000^{\mathrm{a}}\end{array}$ \\
\hline
\end{tabular}


Figure 1. Comparison of Total phenol, Flavanoid, Vitamin C, Anthocyanin,TotalAntioxidant Capacity content of strawberry, promogrent, red grapes, orange, DGT

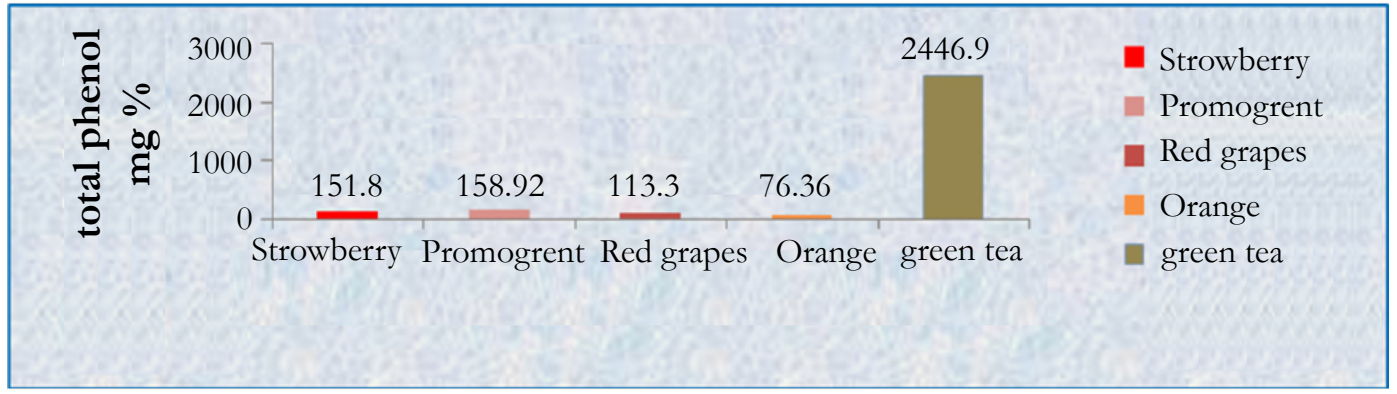
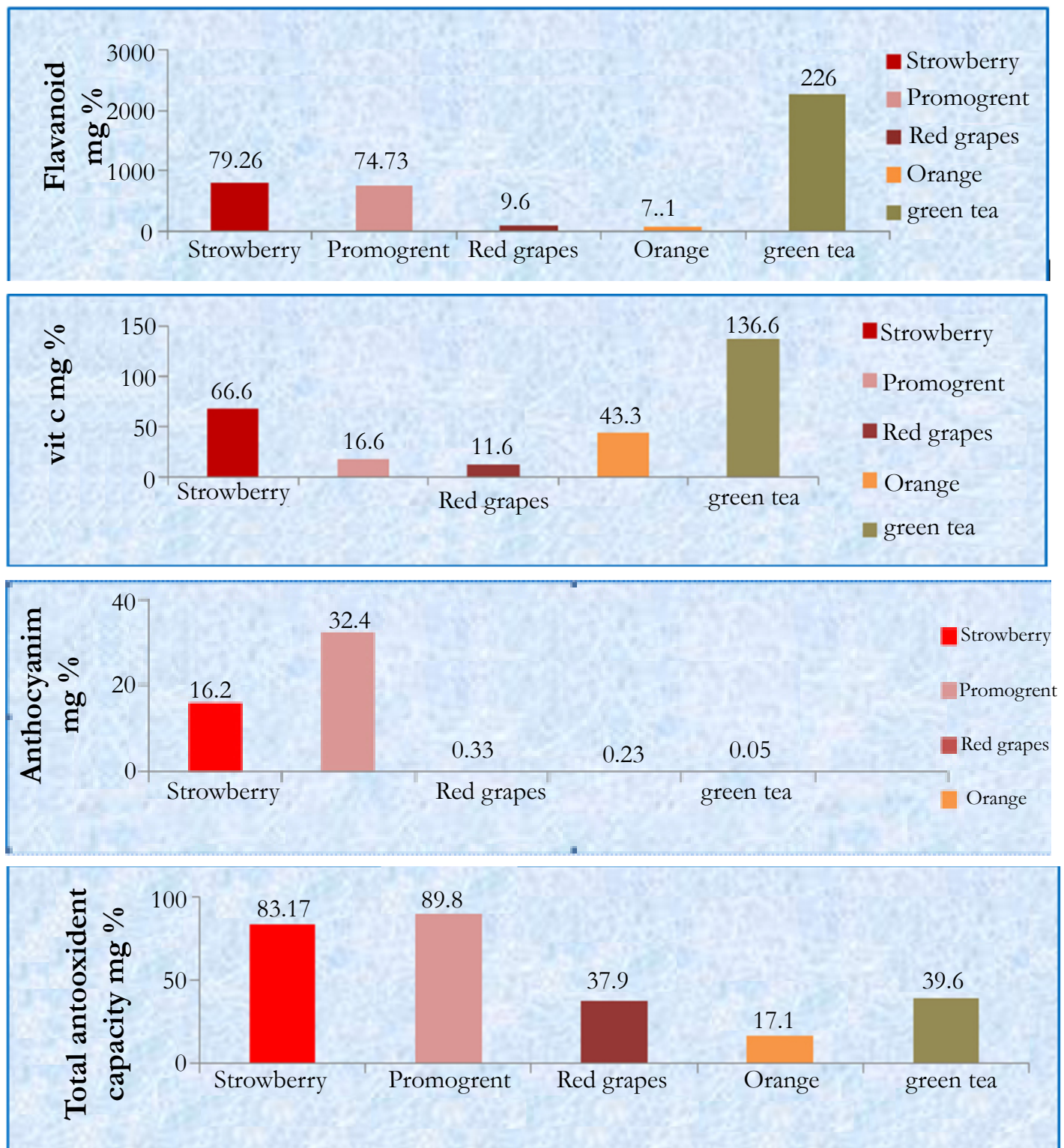

Figure 2. Comperication of sensory score of fruit beverges at different ratio

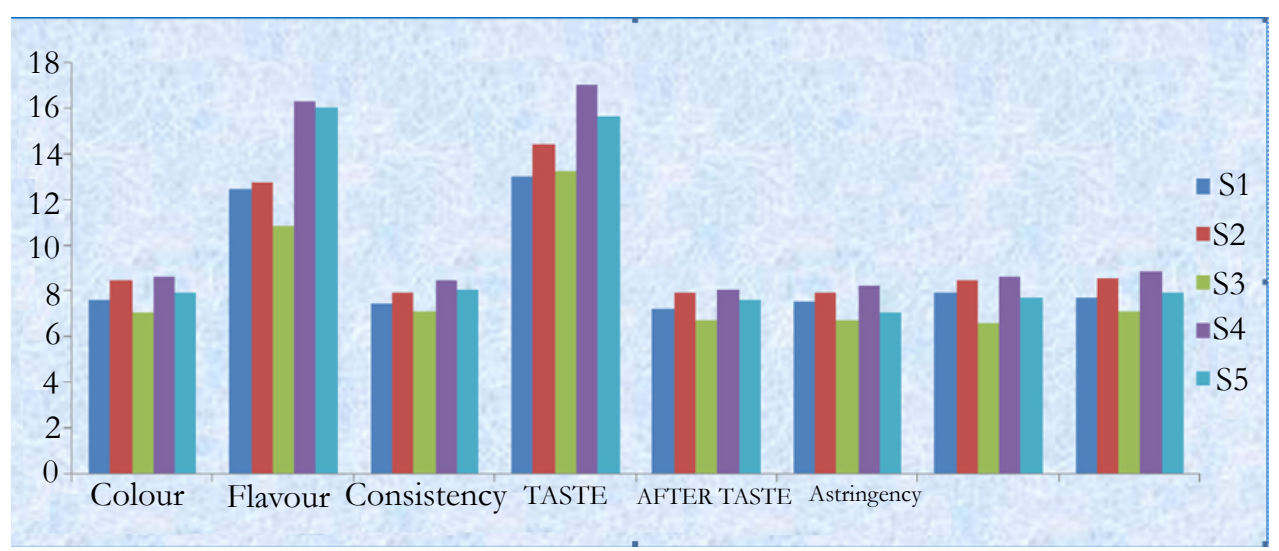


Table 2. Total phenol, Flavanoid, Vitamin C, Anthocyanin, Total Antioxidant Capacity content of Fruit Beverage

\begin{tabular}{|l|l|l|l|l|l|}
\hline Sample & $\begin{array}{l}\text { Total phenol } \\
\mathrm{mg} \%\end{array}$ & $\begin{array}{l}\text { Flavonoid } \\
\mathrm{mg} \%\end{array}$ & Vitamin C mg \% & Anthocyanin $\mathrm{mg} \%$ & Total antioxidant Capacity mg \% \\
\hline Fruit beverages & $\begin{array}{l}282.667 \\
\pm 7.62066\end{array}$ & $\begin{array}{l}44 \\
\pm 4.26654\end{array}$ & $\begin{array}{l}116.667 \\
\pm 1.66667\end{array}$ & $\begin{array}{l}5.493 \\
\pm .10990\end{array}$ & $\begin{array}{l}82.053 \\
\pm 2.87370\end{array}$ \\
\hline
\end{tabular}

Table 3. Sensory score of Fruit beverages at different ratio

\begin{tabular}{|l|l|l|l|l|l|l|l|l|}
\hline $\begin{array}{l}\text { SENSORY } \\
\text { EVALUTION }\end{array}$ & COLOR & FLAVOR & $\begin{array}{l}\text { CONSIS } \\
\text { TENCY }\end{array}$ & TASTE & $\begin{array}{l}\text { AFTER } \\
\text { TASTE }\end{array}$ & $\begin{array}{l}\text { ASTRI } \\
\text { GENCY }\end{array}$ & $\begin{array}{l}\text { AC- } \\
\text { CPETI } \\
\text { BILITY }\end{array}$ & $\begin{array}{l}\text { ADSENT OF } \\
\text { DEFECT }\end{array}$ \\
\hline Sample 1 & $\begin{array}{l}7.6^{\mathrm{ab}} \\
\pm 0.55\end{array}$ & $\begin{array}{l}12.4^{\mathrm{ab}} \\
\pm 2.42\end{array}$ & $\begin{array}{l}7.4^{\mathrm{ab}} \\
\pm 0.77\end{array}$ & $\begin{array}{l}13^{\mathrm{a}} \\
\pm 2.19\end{array}$ & $\begin{array}{l}7.2^{\mathrm{ab}} \\
\pm 0.76\end{array}$ & $\begin{array}{l}7.5^{\mathrm{abc}} \\
\pm 0.87\end{array}$ & $\begin{array}{l}7.9^{\mathrm{b}} \\
\pm 0.69\end{array}$ & $\begin{array}{l}7.7^{\mathrm{ab}} \\
\pm 0.86\end{array}$ \\
\hline Sample 2 & $8.4^{\mathrm{bc}}$ & $12.7^{\mathrm{ab}}$ & $7.9^{\mathrm{ab}}$ & $14.4^{\mathrm{ab}}$ & $7.9^{\mathrm{ab}}$ & $7.9^{\mathrm{bc}}$ & $8.4^{\mathrm{b}}$ & $8.5^{\mathrm{bc}}$ \\
& \pm 16 & \pm 2.61 & \pm 0.79 & \pm 2.26 & \pm 0.63 & \pm 0.69 & \pm 0.55 & \pm 0.73 \\
\hline Sample 3 & $7^{\mathrm{a}}$ & $10.8^{\mathrm{a}}$ & $7.1^{\mathrm{a}}$ & $13.2^{\mathrm{a}}$ & $6.7^{\mathrm{a}}$ & $6.7^{\mathrm{a}}$ & $6.6^{\mathrm{c}}$ & $7.1^{\mathrm{a}}$ \\
& \pm 0.07 & \pm 2.46 & \pm 0.79 & \pm 2.23 & \pm 0.72 & \pm 0.61 & \pm 0.48 & \pm 0.50 \\
\hline Sample 4 & $8.6^{\mathrm{c}}$ & $16.3^{\mathrm{b}}$ & $8.4^{\mathrm{b}}$ & $17^{\mathrm{b}}$ & $8^{\mathrm{b}}$ & $8.2^{\mathrm{c}}$ & $8.6^{\mathrm{b}}$ & $8.8^{\mathrm{c}}$ \\
& \pm 0.55 & \pm 2.17 & \pm 0.40 & \pm 2.15 & \pm 0.72 & \pm 0.59 & \pm 0.29 & \pm 0.36 \\
\hline Sample 5 & $7.9^{\mathrm{abc}}$ & $16^{\mathrm{b}}$ & $8^{\mathrm{ab}}$ & $15.6^{\mathrm{ab}}$ & $7.6^{\mathrm{ab}}$ & $77^{\mathrm{ab}}$ & $7.7^{\mathrm{b}}$ & $7.9^{\mathrm{abc}}$ \\
& \pm 0.57 & \pm 1.82 & \pm 0.60 & \pm 2.07 & \pm 0.82 & \pm 0.47 & \pm 0.54 & \pm 0.42 \\
\hline F- value & $4.00^{* *}$ & $3.59^{* *}$ & $1.83^{\mathrm{NS}}$ & $1.95 \mathrm{NS}$ & $1.76 \mathrm{NS}$ & $2.91^{*}$ & $7.22^{* *}$ & $4.05^{* *}$ \\
& \pm 0.007 & \pm 0.01 & \pm 0.13 & \pm 0.11 & \pm 0.15 & \pm 0.03 & \pm 0.00 & \pm 0.00 \\
\hline
\end{tabular}

found to inhibit reactive oxygen species formation and protect against coronary heart disease, inhibit the development of certain types of cancer, such as breast cancer, and slow tumor growth, help prevent chronic disease.By considering this point an evolution of antioxidant, developed fruit beverage and sensory evolution studies was conducted. The fruit beverage contains large quantities of all the antioxidants which prevent human health problems.

\section{References}

[1]. Amit P, Shailendra B (2011) "Pomegranate Juice Is Potentially Better Than Orange Juice in Improving Antioxidant Function in Elderly Subjects" Inter- natonal Journal of Biochemistry Research \& Review 1: 14-23.

[2]. Use of Fruits in the form downloaded from www.suu.edu/faculty/.../FruitRead.PDF.

[3]. Patrizia R, Francesco V, Claudio G, Simona G, Antonella B, et al. (2005) "effect of blood orange juice intake on antioxidant bioavailability and on the different markers related to oxidative stress" journal of agricultural and food chemistry 53:941-947.

[4]. FRUIT JUICE NUTRITION \& HEALTH - SUMMARY. http://www citrusbr.com/download/FRUIT_JUICE_NUTRITION_ShortPaper_a+a_ final_Korrek.pdf

[5]. Patrick E. D, Thomas M. C, Michael G. C Jr, Joe A. V (2010) Vitamin Enhanced Waters and Polyphenol Rich Beverages Analyzed for Antioxidant Capacity and Antioxidants/Calorie 2:1290-1296

[6]. Wada L, Ou B. (2002) "Antioxidant activity and phenolic content of Oregon cranberries.” J. Agric Food Chem 50:3495-3500.

[7]. Ronald L, Guohua C (2000) "Antioxidant phytochemicals in fruits and vegetables: Diet and Health implications” Hortscience. 35(4). 